\title{
Ginsenoside Rb1 Preconditioning Enhances eNOS Expression and Attenuates Myocardial Ischemia/Reperfusion Injury in Diabetic Rats
}

\author{
Rui Xia, Bo Zhao, Yang Wu, Jia-Bao Hou, Li Zhang, Jin-Jin Xu, and Zhong-Yuan Xia \\ Department of Anesthesiology, Renmin Hospital of Wuhan University, Wuhan, Hubei 430060, China \\ Correspondence should be addressed to Zhong-Yuan Xia, xiazhongyuan2005@yahoo.com.cn
}

Received 18 December 2010; Revised 3 July 2011; Accepted 22 July 2011

Academic Editor: Leon Spicer

Copyright $\odot 2011$ Rui Xia et al. This is an open access article distributed under the Creative Commons Attribution License, which permits unrestricted use, distribution, and reproduction in any medium, provided the original work is properly cited.

\begin{abstract}
Diabetes mellitus is associated with decreased NO bioavailability in the myocardium. Ginsenoside Rb1 has been shown to confer cardioprotection against ischemia reperfusion injury. The aim of this study was to investigate whether Ginsenoside Rb1 exerts cardioprotective effects during myocardial ischemia-reperfusion in diabetic rats and whether this effect is related to increase the production of NO via enhancing eNOS expression in the myocardium. The myocardial I/R injury were induced by occluding the left anterior descending artery for $30 \mathrm{~min}$ followed by $120 \mathrm{~min}$ reperfusion. An eNOS inhibitor L-NAME or Rb1 were respectively administered $25 \mathrm{~min}$ or $10 \mathrm{~min}$ before inducing ischemia. Ginsenoside Rb1 preconditioning reduced myocardial infarct size when compared with I/R group. Ginsenoside Rb1 induced myocardial protection was accompanied with increased eNOS expression and NO concentration and reduced plasma CK and LDH $(P<0.05)$. Moreover, the myocardial oxidative stress and tissue histological damage was attenuated by Ginsenoside Rb1 $(P<0.05)$. L-NAME abolished the protective effects of Ginsenoside $\mathrm{Rb} 1$. It is concluded that Ginsenoside Rb1 protects against myocardium ischemia/reperfusion injury in diabetic rat by enhancing the expression of eNOS and increasing the content of NO as well as inhibiting oxidative stress.
\end{abstract}

\section{Introduction}

Diabetes mellitus has long been identified as the leading cause of the development of ischemic heart disease within the diabetic population [1]. Many epidemiological findings unanimously support the fact that cardiovascular diseases (CVD) are the primary mortality factor among diabetic patients [2]. The diabetic state is associated with increased oxidative stress, and the hyperglycemia further stimulates the production of advanced glycosylated end products, which increase microvascular permeability. Moreover, the abnormal lipid metabolism increases oxidized low-density lipoprotein (ox-LDL) formation, resulting in oxidative stress which exacerbates myocardial and vascular endothelial cell insult due to myocardial ischemia/reperfusion injury. Hearts from diabetic subjects are less resistant to ischemic insults $[3,4]$. We have recently demonstrated that postischemic myocardial infarct sizes were significantly larger in diabetic rats 8 weeks after STZ-injection as compared to non-diabetic controls [5], and we have found that Rb1 can confer cardioprotection in diabetic rats. However, the mechanism by which $\mathrm{Rb} 1$ confers cardioprotection in diabetes has not been explored. Therefore, in the current study, we explored whether or not enhancing nitric oxide bioavailability plays a critical role in Rb1-mediated cardioprotection in diabetic subjects.

Nitric oxide (NO) is a fundamental endothelium-derived relaxing factor (EDRF), which at physiological concentration possesses vasodilatory properties inhibits leukocyte and platelet adherence, suppresses smooth muscle cell hyperplasia [6], and is also beneficial in limiting myocardial ischemia/ reperfusion $(\mathrm{MI} / \mathrm{R})$ injury. Numerous studies have already demonstrated the cardioprotective effects of NO during ischemia/reperfusion events $[7,8]$. However, in patients with long-standing diabetes, the availability of $\mathrm{NO}$ decreases progressively [9]; in addition, eNOS expression is reduced as well as mediators for the synthesis of NO. Hence, such situations aggravate vascular endothelial dysfunction and 
atherosclerosis in diabetes $[10,11]$. In animal studies, it has been found that eNOS provides protection to some extent against I/R injury to extracorporeal hearts [12], and bioavailability of $\mathrm{NO}$ and availability of eNOS decrease I/R injury and promote reperfusion of myocardial infarction and after-heart failure in left ventricular function recovery [13].

Ginsenoside Rb1 is the main bioactive component of Ginseng and Shenfu injection. It has various beneficial effects on the cardiovascular system, such as scavenging free radicals, blocking calcium overinflux into neurons, inhibiting $\mathrm{Na}^{+}$channel activities, improving energy metabolism, and preserving the structural integrity of the neurons, among many others [14]. Recent studies have demonstrated that Ginsenoside Rb1 has protective effects against myocardial ischemic injury in nondiabetic animals [15] and the molecular mechanism of this beneficial effect is still unidentified.

The present study aims to determine whether Ginsenoside $\mathrm{Rb} 1$ preconditioning can protect against $\mathrm{I} / \mathrm{R}$ injury in diabetic rats by enhancing the expression of eNOS and increasing the content of $\mathrm{NO}$ as well as inhibiting oxidative stress.

\section{Materials and Methods}

2.1. Experimental Animals. The experimental procedures and protocols used in this research work were approved by the Animal Use Committee of Wuhan University. Healthy male adult Sprague-Dawley (SD) rats (weighing 220 280 g), procured from the Experimental Animal Centre of Wuhan University. After one week of adaption period, they were kept fasting for $12 \mathrm{hr}$ and injected with streptozotocin (STZ; Sigma, USA; pH 4.5) intraperitoneally at a dosage of $65 \mathrm{mg} / \mathrm{kg}$. Five days after STZ injection, the rats were kept fasting for $5 \mathrm{hr}$, and the blood samples from the tails were collected and fasting blood sugar was measured using SureStrep glucometer (Johnson \& Johnson Company). Rats with blood glucose level $\geq 16.7 \mathrm{mmol} / \mathrm{L}(300 \mathrm{mg} / \mathrm{L})$ were considered diabetic models [16].

2.2. Myocardial Ischemia: Reperfusion Model. After a 12 hour period of fasting, the animals were anesthetized with $3 \%$ pentobarbital sodium injection $(50 \mathrm{mg} / \mathrm{kg}$ ) administered intraperitoneally and then fixed on the operating table for surgical procedures. ECG electrodes were placed subcutaneously in both front limbs and left back limb and lead II was continuously monitored. A 14-gauge angiocatheter was inserted into the trachea through a midline neck incision and then connected to a volume-controlled ventilator (DW-2000, Jiapeng Keji, Shanghai, China). The left femoral vein was catheterized for intravenous administration of drugs. The physiological saline or drugs (Ginsenoside Rb1, L-NAME) was slowly administered through the femoral catheter. Heparin $(500 \mathrm{U} / \mathrm{kg})$ was administered intravenous and the right carotid artery was catheterized for the continuous monitoring of mean arterial pressure (MAP) and for blood sampling. The electrocardiogram and blood pressure signal was displayed on an M3 monitor (Siemens, Germany). A left thoracotomy in the fourth intercostal space was performed and the pericardium was opened. A 6-0 silk suture was passed with a tapered needle under the left anterior descending coronary artery, $2 \mathrm{~mm}$ from the tip of the left auricle. The ends of the suture were threaded through a piece of plastic tubing, forming a snare that occluded the artery when tightened and clamped. The coronary artery was occluded for $30 \mathrm{~min}$ and then allowed reperfusion (by releasing the clamp) for $2 \mathrm{hr}$.

2.3. Experimental Protocol. The diabetic rats were randomly assigned to five groups, and each group 8 rats: group 1, shamoperated rats (Sham); group 2, ischemia/reperfusion group (30 min of myocardial ischemia and $120 \mathrm{~min}$ of reperfusion, $\mathrm{I} / \mathrm{R}$ ); group 3, Ginsenoside Rb1 preconditioning group, Ginsenoside Rb1 40 mg/kg was administered intravenously 10 min before coronary ischemia and reperfusion (Rb1) [14]; group 4, L-NAME + I/R group, L-NAME was given at a dose of $10 \mathrm{mg} / \mathrm{kg} 25 \mathrm{~min}$ before ischemia (L-NAME) [17]; group 5, L-NAME + Ginsenoside Rb1-treated group (LNAME $+\mathrm{Rb} 1)$. In the second set of experiments, seven similar experimental groups of rats $(n=4$ in each group) were subjected to the same experimental procedures. At the end of reperfusion, the animals were sacrificed by an intravenous injection of $10 \%$ potassium chloride solution, and myocardial samples were collected from ischemic left ventricle regions for protein analysis. All animals survived after ischemia reperfusion until being terminated at the completion of the experiment. The samples were frozen in liquid nitrogen, and stored at $-80^{\circ} \mathrm{C}$ for later analysis.

2.4. Determination of the Area at Risk and Infarct Size. At the end of the $2 \mathrm{hr}$ of reperfusion, the rats were given heparin ( $1 \mathrm{U} / \mathrm{g}$, intraperitoneally). The coronary artery was reoccluded, and $1 \mathrm{~mL}$ of $1.5 \%$ solution of Evans blue dye was injected via the left femoral vein to identify the ischemic risk area. $2 \mathrm{~mL}$ of $10 \%$ potassium chloride solution was injected into the femoral vein to stop the heart, and then, the heart was excised. The presence of Evans blue dye indicated nonischemic area and its absence indicated area at risk (AAR). After resecting the right ventricle, the left ventricle (LV) was cut into five transverse slices from the apex to the base. The slices were incubated in $1 \%$ triphenyltetrazolium chloride (TTC) solution at $37^{\circ} \mathrm{C}$ for $25 \mathrm{~min}$, and they were then photographed with a digital camera (Cannon, PowerShot AS1000, Japan) and weighed. For each slice, the total LV area, the area lacking Evans blue staining (AAR) and the area lacking TTC staining (infarct area, IA) were determined using a light electron microscope (Olympus, Japan). The ratios of IA/AR and AAR/LV were calculated for each slice and then summed over all slices.

\subsection{Detection of Plasma Creatine Kinase (CK) and Lactate} Dehydrogenase $(L D H)$. The cardiac damage was evaluated by measuring plasma creatine kinase (CK) and lactate dehydrogenase (LDH). The blood samples were drawn from the right carotid artery at the end of reperfusion period. The plasma concentrations of $\mathrm{CK}$ and $\mathrm{LDH}$ were measured by automatic biochemical analyzer (AU-2700, Olympus, Japan). 
2.6. Histopathological Examination. At the end of the experiments, the formalin-fixed, paraffin-embedded sections of myocardial tissues were fixed in $10 \%$ buffered formalin and sections were prepared from paraffin-embedded tissues. The level of histological tissue injury was assessed by haematoxylin and eosin (H\&E) staining using light microscopy (400x magnification).

2.7. Immunohistochemical Staining for eNOS. The expression of eNOS was determined by immunohistochemistry. Paraffin-embedded left ventricular tissue blocks were sectioned at $3 \mu \mathrm{m}$. The sections were deparaffinized, rehydrated, treated with target retrieval buffer, blocked with 3\% hydrogen peroxide, washed with phosphate-buffered saline (PBS), and blocked with 5\% normal goat serum in PBS for $30 \mathrm{~min}$. The sections were then incubated overnight with the polyclonal antiendothelial nitric oxide synthase (eNOS) antibody (rabbit antiendothelial nitric oxide synthase, 1:500, pH 7.2, with $0.1 \%$ bovine serum albumin (Boster Bio-Tech, Wuhan, China), followed by biotin-conjugated secondary antibody at $1: 1000$ dilutions. Finally, the sections were incubated with avidin-biotin complex kit (Boster Bio-Tech, Wuhan, China) and detected by using a diaminobenzidine (DAB) reagent (Boster Bio-Tech Wuhan, China). The slides were examined in 400-fold magnification by light microscopy (Olympus BX50 Microphotographic System, Japan). For each animal, three random tissue sections (five fields per section) were examined. Quantitative immunohistochemical assessments for myocardial eNOS expression were performed as previously described. A mean optical density (OD), which related to immunohistochemical staining intensity, was measured by image cytometry with HIPAS-2000 image analysis software (Qianli Technical Imaging, Wuhan China).

2.8. Determination of SOD and MDA. The myocardial oxidative stress contents were assayed by the measurement of SOD and MDA. At the end of reperfusion, the myocardial supernatant was isolated from ischemia heart tissue samples by centrifugation at $4000 \mathrm{rpm}$ for $10 \mathrm{~min}$ at $4^{\circ} \mathrm{C}$. The MDA level in the supernatant was determined by the measurement of the thiobarbituric acid (TBA) reaction using a commercial kit (Jiancheng Biological, Nanjing, China) [18]. And the SOD activity in the supernatant was evaluated by inhibition of nitroblue tetrazolium (NBT) reduction by $\mathrm{O}_{2}^{-}$generated by the xanthine/xanthine oxidase system using a commercial kit (Jiancheng Biological, Nanjing, China) [19].

2.9. Assay of NO Production. The level of NO in myocardial tissue was measured by the Griess method and according to the indication on the $\mathrm{NO}$ assay kit (Jiancheng Biological, Nanjing, China). The myocardium was lysed and centrifuged and then $50 \mu \mathrm{L}$ of Griess reagent was added to $50 \mu \mathrm{L}$ of supernatant. Nitrite concentration was determined by a spectrophotometer (721-100; Tianpu Analytical Instrument Co., Shanghai, China) at $550 \mathrm{~nm}$ from a standard curve $(0-$ $100 \mu \mathrm{mol} / \mathrm{L}$ ) derived from $\mathrm{NaNO}_{2}$.

2.10. Statistical Analysis. Values were expressed as means \pm SEM. All biochemical parameters including plasma LDH,
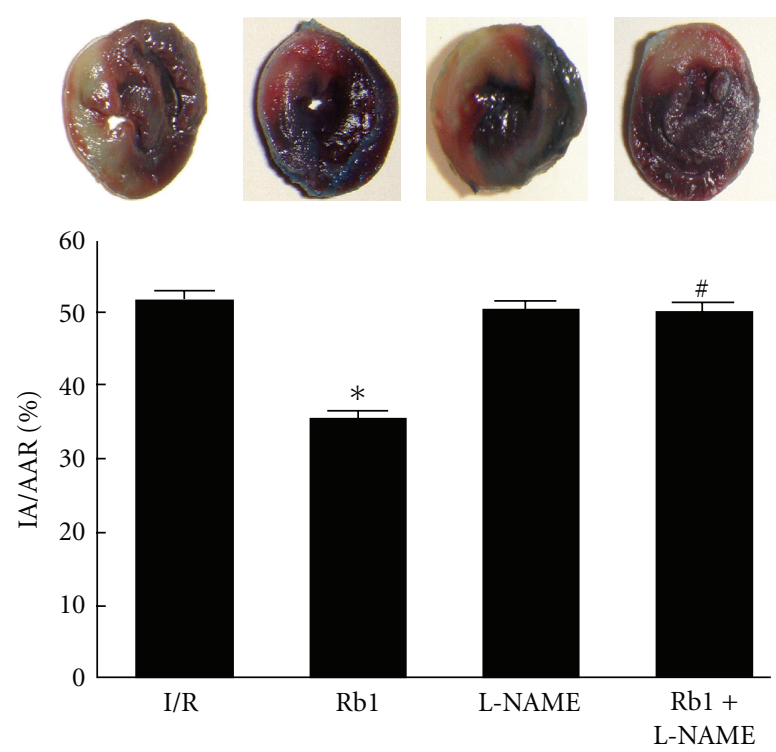

FIGURE 1: Infarct size expressed as percent of the area at risk in rats subjected to $30 \mathrm{~min}$ ischemia and $2 \mathrm{~h}$ of reperfusion. The bluestained areas represent nonischemic tissue and red-stained areas represent the area at risk. Pale areas indicate infarct areas. Results are expressed as mean \pm SEM. $n=6,{ }^{*} P<0.01$ versus I/R group, ${ }^{\#} P<0.01$ versus $\mathrm{Rb} 1$ group.

CK, SOD, and MDA were assayed in duplicate. Therefore, these data are themselves means of duplicate assays. Statistical analysis was performed using SPSS 13.0 for Windows software. The statistical significance was determined by ANOVA. A value of $P<0.05$ was considered to be statistically significant.

\section{Results}

3.1. Baseline and Model Characteristics. For each diabetic animal model, a 4-hour fasting plasma glucose (FPG) was measured every 14 days. The diabetic rats with fasting plasma glucose concentrations $\geq 16.7 \mathrm{mmol} / \mathrm{L}$ showed signs of polydipsia, polyphagia and polyuria (Table 1).

3.2. Infarct Size Assessment of the Operated Groups. The ratio of area at risk of left ventricular mass (AAR/LV) did not differ significantly among the groups. In I/R group, the infarct size was $51.7 \pm 4.34 \%$ of the area at risk (IS/AAR). Pretreatment with Ginsenoside $\mathrm{Rb} 1$ yielded an infarct size of $36.9 \pm 2.34 \%$ $(P<0.05$ versus $\mathrm{I} / \mathrm{R}$ group $)$. Administration of L-NAME or $\mathrm{I} / \mathrm{R}+\mathrm{Rb} 1+\mathrm{L}-\mathrm{NAME}$ did not affect infarct size $(P>0.05$ versus I/R group). Pretreatment with L-NAME hindered the cardioprotective effect of Ginsenoside Rb1 preconditioning $(P<0.05$ versus $\mathrm{Rb} 1$ group) (Figure 1$)$.

\subsection{Effects of Ginsenoside Rb1 on Plasma CK and LDH. To} investigate whether Ginsenoside Rb1 limited cardiomyocyte necrosis, the activity of serum CK and LDH were measured at the end of reperfusion period. Comparing with Sham group, serum CK and LDH of I/R group were markedly 
TABLE 1: The fasting plasma glucose and body weight of diabetic rats $(n=60)$.

\begin{tabular}{lccccc}
\hline Parameter & $5 \mathrm{~d}$ & $2 \mathrm{~W}$ & $4 \mathrm{~W}$ & $6 \mathrm{~W}$ & $8 \mathrm{~W}$ \\
\hline FPG $(\mathrm{mmol} / \mathrm{L})$ & $19.9 \pm 2.4$ & $23.0 \pm 2.4$ & $22.9 \pm 1.9$ & $22.4 \pm 3.8$ & $25.2 \pm 2.8$ \\
BW $(\mathrm{g})$ & $258.5 \pm 35.4$ & $273.6 \pm 23.2$ & $283.6 \pm 28.4$ & $266.0 \pm 21.5$ & $242.8 \pm 22.3$ \\
\hline
\end{tabular}
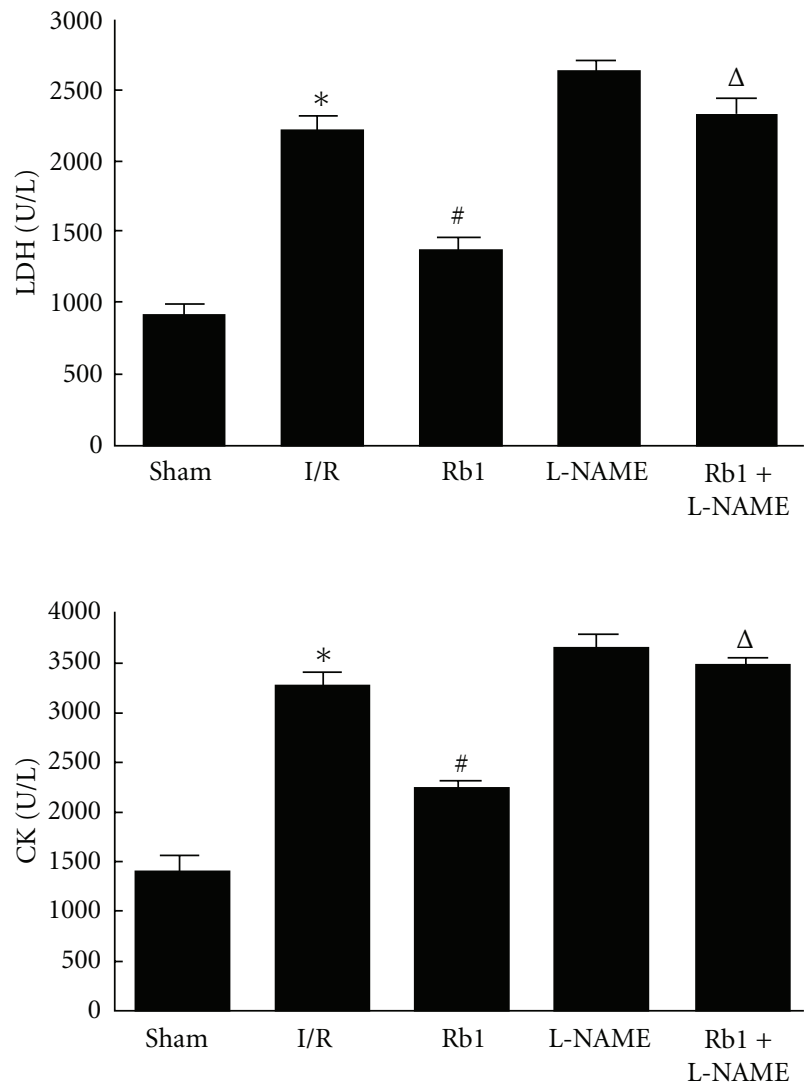

FIGURE 2: Effects of Rb1 with or without L-NAME pretreatment on plasma levels of $\mathrm{CK}, \mathrm{LDH}$ after $30 \mathrm{~min}$ of myocardial ischemia and $120 \mathrm{~min}$ of reperfusion. Results are expressed as mean \pm SEM. ${ }^{*} P<0.01$ versus Sham group, ${ }^{\#} P<0.01$ versus I/R group, ${ }^{\Delta} P<$ 0.01 versus $\mathrm{Rb} 1$ group.

increased (both $P<0.01$ ). After preconditioning with Ginsenoside Rb1, the CK and LDH levels were significantly lower compared with the I/R group (both $P<0.05$ ). Moreover, pretreatment with L-NAME completely abolished Ginsenoside Rb1-induced decrease in plasma CK, LDH (Figure 2).

3.4. Effects of Ginsenoside Rb1 on Myocardial SOD and MDA Levels. To examine whether Ginsenoside Rb1 decreased the diabetic myocardial oxidative stress, SOD and MDA levels in the ischemia heart tissue were measured. Comparing with Sham group, MDA level in I/R group was markedly increased, while SOD activity was lower $(P<0.05)$. After preconditioning with Ginsenoside Rb1, MDA levels in Rb1 and Rb1 + L-NAME groups were decreased significantly
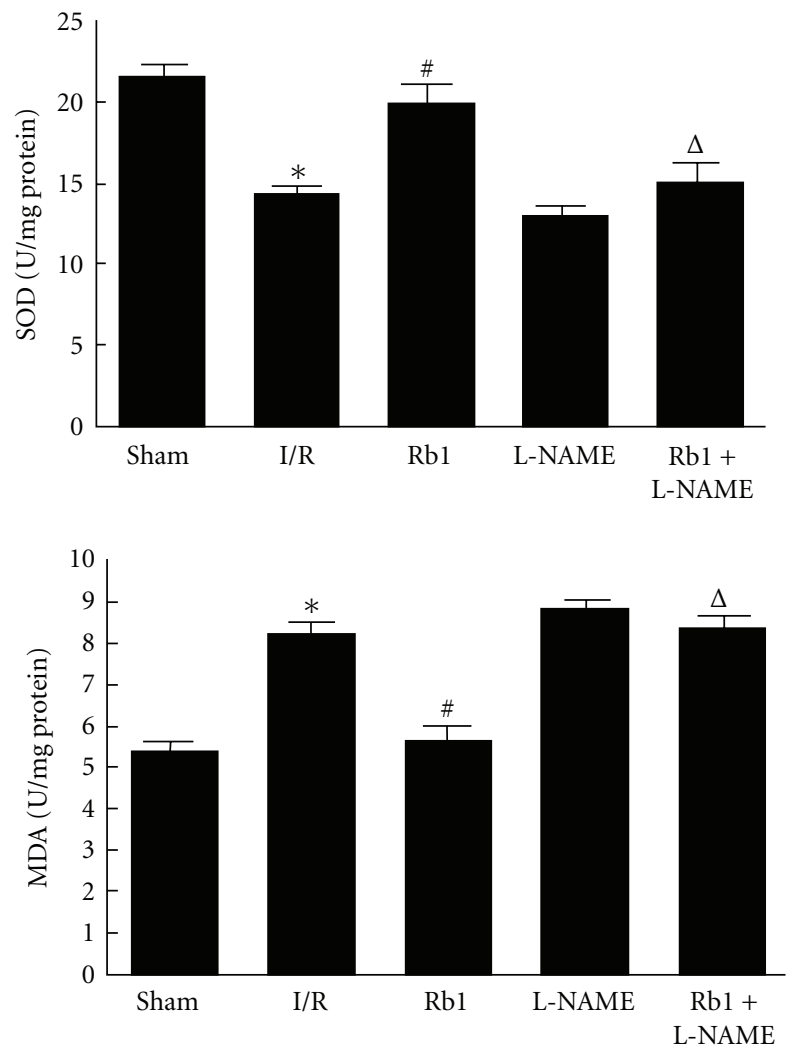

Figure 3: Effect of Ginsenoside Rb1 preconditioning on SOD and MDA. Results are expressed as mean \pm SEM. ${ }^{*} P<0.01$ versus Sham group, ${ }^{\#} P<0.05$ versus I/R group, ${ }^{\Delta} P<0.05$ versus $\mathrm{Rb} 1$ group.

compared with the I/R group, while the activity of SOD were higher $(P<0.05)$, (Figure 3$)$.

\subsection{Effect of Ginsenoside Rb1 on Myocardial NO Production.} To further investigate the biochemical events associated with Ginsenoside Rb1 pretreatment, NO production in ischemic heart was evaluated. NO production, which was indicated as the nitrite formation, was found to be decreased in I/R group. We found that Ginsenoside Rb1 induced a significant raise in nitrite level $(P<0.05)$; however, this effect of elevated nitrite formation was inhibited with the presence of L-NAME $(P<$ 0.05), (Figure 4).

3.6. Expression of eNOS in the Diabetic Heart. As shown in Figure 5, a very small amount of eNOS was detected in myocardial tissue of the Sham group. Significantly reduced expression of eNOS was observed in group I/R $(P<0.05$, 


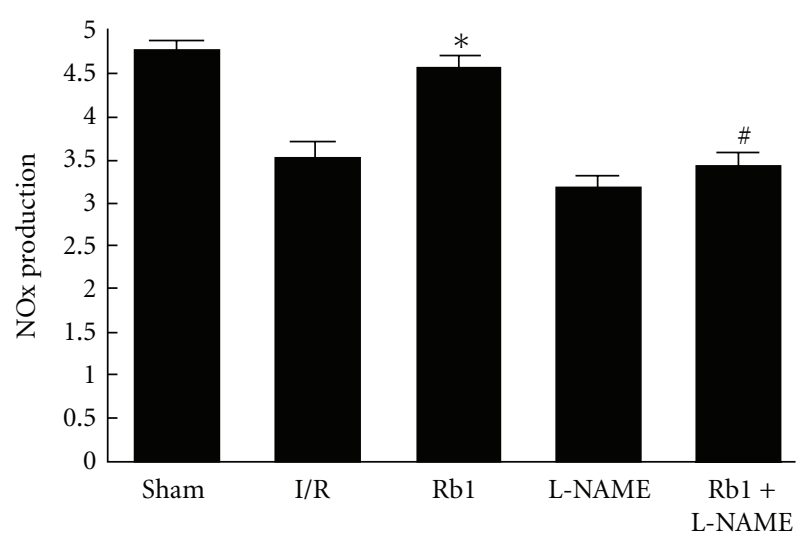

Figure 4: Effect of Ginsenoside Rb1 preconditioning on NO production. Results are expressed as mean \pm SEM. ${ }^{*} P<0.05$ versus I/R group, ${ }^{\#} P<0.01$ versus $\mathrm{Rb} 1$ group.

versus Sham). When compared with group I/R, group $\mathrm{Rb} 1$ had significantly higher expression of eNOS $(P<0.05)$. Pretreatment with L-NAME almost completely abolished the induction of the expression of eNOS $(P<0.05$, L-NAME + Rbl versus Rbl group).

3.7. Heart Histopathologic Changes. The histopathological changes in the ischemic heart tissue at the end of reperfusion were assessed by standard $\mathrm{H} \& \mathrm{E}$ staining. As shown in Figure 6, the myocardium of Sham group maintained normal tissue structure and shape, and the myocardial fibers were arranged in an orderly manner. I/R group showed acute heart injury characterized by areas of necrosis, neutrophilic inflammation, myocardial, and interstitial edema. The myocardial fibers were partially ruptured and lysed. Rb1 group revealed markedly reduced neutrophilic inflammation and interstitial edema with preservation of myocardium compared with I/R group. More importantly, L-NAME was administered prior to Rb1, the destruction of heart tissue was more severely as compared with I/R group.

\section{Discussion}

In this study, Ginsenoside Rb1 preconditioning limited the heart infarct size and MDA concentrations, while increasing SOD activity and attenuating cellular damage. The protective effects induced by Ginsenoside Rb1 preconditioning were related to the expression of eNOS and NO. These protective effects were blocked by an eNOS inhibitor (L-NAME), suggesting that eNOS activity and endothelium-derived NO mediated the protective effects of Ginsenoside Rb1 pretreatment.

Several mechanisms have been proposed for the oxidative damage during chronic hyperglycemia, such as mitochondrial reactive oxygen species (ROS) overproduction [20], glucose auto-oxidation [21], synthesis of AGEs [22], and hyperglycemia reduced NO availability. Endothelial dysfunction has been documented in various forms of diabetes and even in prediabetic individuals $[23,24]$. The pathogenesis of this endothelial dysfunction involves many components including increased polyol pathway flux, altered cellular redox state, increased formation of diacylglycerol, and the subsequent activation of specific protein kinase $\mathrm{C}$ isoforms, and accelerated nonenzymatic formation of AGEs. There are many clinical and experimental evidences suggesting that increased sympathetic activities, activated cardiac reninangiotensin system, myocardial ischemia/functional hypoxia, and elevated circulating levels of glucose result in oxidative stress in cardiovascular system of diabetic subjects. Oxidative stress associated with an impaired antioxidant defense status may play a critical role in subcellular remodeling, calciumhandling abnormalities, and subsequent diabetic cardiomyopathy [25]. A clinical study indicated that systemic oxidative stress occurred during cardiopulmonary bypass and postcardiopulmonary bypass periods and also in patients with diabetes mellitus, and the systemic oxidative stress was higher [26]. So, we hypothesized that Ginsenoside Rb1 pretreatment may attenuate myocardial ischemia reperfusion injury through an increase in the activity of eNOS, enhancing the availability of $\mathrm{NO}$, and decreasing the oxidative stress.

NO released from endothelial cells, is a pivotal vasoprotective molecule, which maintains normal vascular tone, regulates leukocyte-endothelial cell interactions, inhibits platelet aggregation, limits smooth muscle cell proliferation, and may affect cardiac myocyte function, neutrophil activation, and free-radical production $[11,27,28]$. Previous studies clearly demonstrated that the deficiency of eNOS exacerbates myocardial I/R injury [29], and myocardial ischemia and reperfusion are associated with marked impairment of endothelium-dependent coronary relaxation, reduced contractility, and cardiac arrhythmias. In many studies, reperfusion injury has been attributed to a reduced formation or activity of NO because the NO precursor, L-arginine, NO donors of different chemical structures, and interventions resulting in stimulated $\mathrm{NO}$ production involving endothelial receptors have all been found to protect the heart [30]. Moreover, the overexpression of eNOS, the administration of NO donors, and inhaled NO gas therapy all significantly protect the myocardium [31]. Yang et al. [32] also showed that NO released by endothelial NO synthase (eNOS) is important in blood pressure regulation and also has an impact on cardiac function and remodeling.

In addition, there are evidences strongly suggesting that Ginsenoside Rb1 bears various beneficial effects on the cardiovascular system. In cell culture model, Ginsenoside Rb1 enhanced NO production and the expression of eNOS mRNA in TNF- $\alpha$ stimulated HUVEs. [33]. In vitro, Ginsenoside Rb1 has been shown to reduce homocysteine-induced endothelial dysfunction and free radical production as well as eNOS downregulation in porcine coronary arteries [34-36]. Study has shown that Ginsenoside Rb1 significantly inhibited cardiocyte apoptosis and reduced ischemia-reperfusion in rats [15]. Recent studies demonstrated that administration of Ginseng berry significantly improved systemic insulin sensitivity and glucose homeostasis in the rats. Others have shown that the Ginsenoside compounds attenuated inhibitory inflammatory response after cardiopulmonary bypass in patients with congenital heart diseases, which played an 

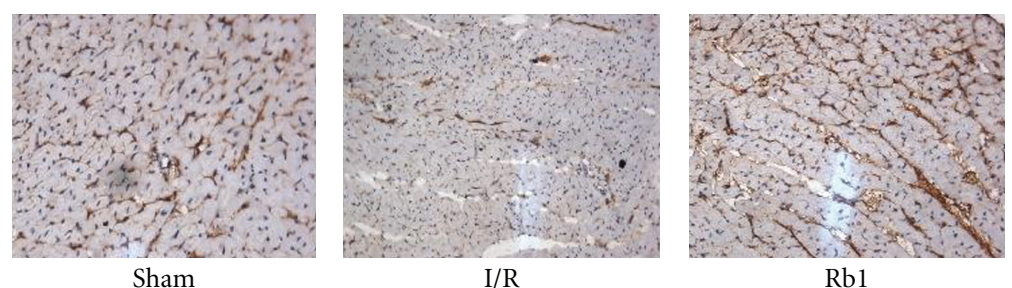

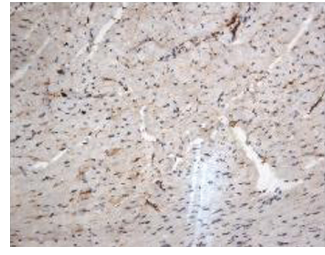

L-NAME

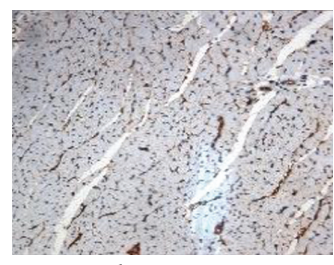

$\mathrm{Rb} 1+\mathrm{L}-\mathrm{NAME}$

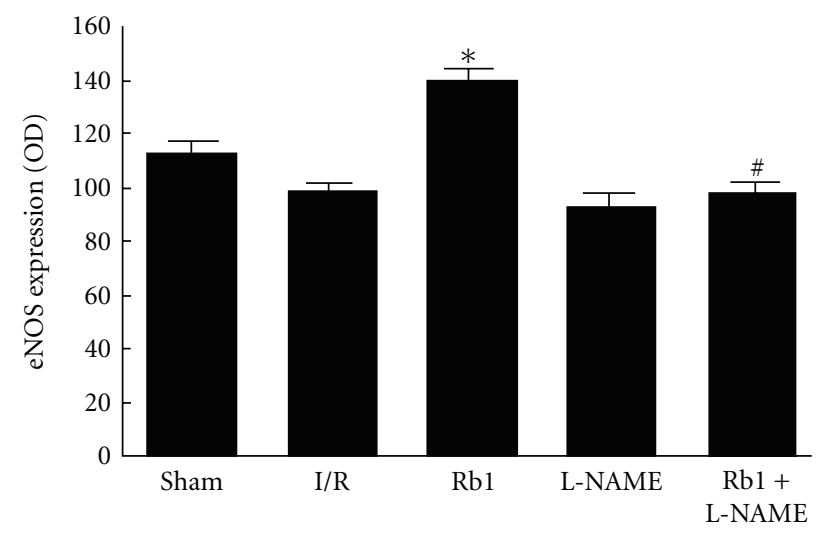

Figure 5: Effect of Ginsenoside Rb1 preconditioning on the production of NOx (nitrite and nitrate, metabolites of nitric oxide (NO)). Results are expressed as mean \pm SEM. ${ }^{*} P<0.01$ versus I/R group, ${ }^{\#} P<0.01$ versus Rb1 group.

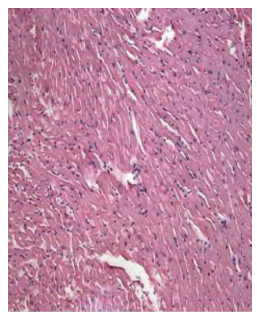

Sham

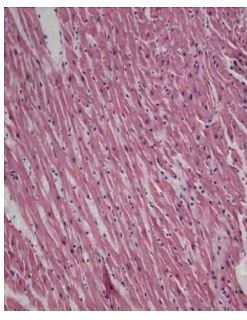

$\mathrm{I} / \mathrm{R}$

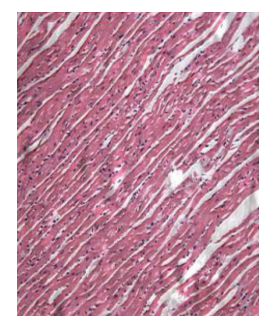

$\mathrm{Rb} 1$

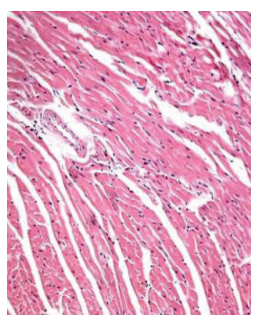

L-NAME

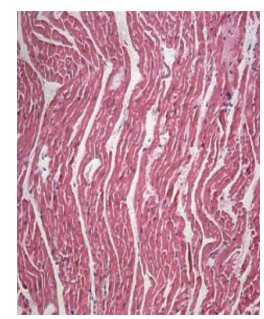

$\mathrm{Rb} 1+\mathrm{L}-\mathrm{NAME}$
Figure 6: Histopathological slides of the diabetic rats heart tissue (magnification, $\times 400$ ).

important role in protecting the heart from ischemicreperfusion injury [37]. Additional researches demonstrated that Ginsenoside plays a significant role in antihyperglycemic action [38]. Therefore, it was important to determine whether pretreatment with Ginsenoside Rb1 would reduce myocardial infarction after ischemia reperfusion events. In addition, a previous study showed that Ginsenoside Rb1, a major active component of Ginseng, could actually induce NO production and regulate acute eNOS activation in human aortic endothelial cells [39].

In this study, by using an in vivo rat model, we found that Ginsenoside Rb1 preconditioning significantly decreased infarct size and plasma $\mathrm{CK}, \mathrm{LDH}$ after $30 \mathrm{~min}$ of regional myocardial ischemia and 120 min of reperfusion, and Ginsenoside Rb1 pretreatment attenuated the increase in necrosis, neutrophilic inflammation respond, and myocardial and interstitial edema. The myocardial MDA level, reflecting the lipid peroxide and oxidative stress, was also significantly suppressed in Rb1 group, while the SOD activity was increased. Ginsenoside Rb1 upregulated eNOS and NO contents, while eNOS inhibitor (L-NAME) reversed the protective effect of Ginsenoside Rbl pretreatment. Therefore, our study demonstrated the protective effect of Ginsenoside Rb1 pretreatment, at least in part, by eNOS and NO. Certain limitations of the present study need to be noted. The current study did not investigate mRNA and protein expressions of eNOS. This will be the goal of future study. 
In summary, our study suggests that Ginsenoside Rb1 preconditioning attenuated the severity of heart injury induced by myocardial ischemia/reperfusion injury in diabetic rats. The protective effect of Ginsenoside Rb1 preconditioning was mediated in part through the induction of endogenous NO released by eNOS.

\section{References}

[1] A. Tsang, D. J. Hausenloy, M. M. Mocanu, R. D. Carr, and D. M. Yellon, "Preconditioning the diabetic heart: the importance of Akt phosphorylation," Diabetes, vol. 54, no. 8, pp. 23602364, 2005.

[2] A. Cerghizan, C. Bala, C. Nita, and N. Hancu, "Practical aspect of the control of cardiovascular risk in type 2 diabetes mellitus and the metabolic syndrome," Experimental and Clinical Cardiology, vol. 12, no. 2, pp. 83-86, 2007.

[3] S. M. Donahoe, G. C. Stewart, C. H. McCabe et al., "Diabetes and mortality following acute coronary syndromes," Journal of the American Medical Association, vol. 298, no. 7, pp. 765-775, 2007.

[4] Y. Zhang, L. Wei, D. Sun et al., “Tanshinone IIA pretreatment protects myocardium against ischaemia/reperfusion injury through the phosphatidylinositol 3-kinase/Akt-dependent pathway in diabetic rats," Diabetes, Obesity and Metabolism, vol. 12, no. 4, pp. 316-322, 2010.

[5] Y. Wu, Z. Y. Xia, J. Dou et al., "Protective effect of ginsenoside Rb1 against myocardial ischemia/reperfusion injury in streptozotocin-induced diabetic rats," Molecular Biology Reports, pp. 1-9, 2010.

[6] A. A. Bulhak, P. O. Sjöquist, C. B. Xu, L. Edvinsson, and J. Pernow, "Protection against myocardial ischaemia/reperfusion injury by PPAR- $\alpha$ activation is related to production of nitric oxide and endothelin-1," Basic Research in Cardiology, vol. 101, no. 3, pp. 244-252, 2006.

[7] A. V. Gourine, A. A. Bulhak, A. T. Gonon, J. Pernow, and P. O. Sjöquist, "Cardioprotective effect induced by brief exposure to nitric oxide before myocardial ischemia-reperfusion in vivo," Nitric Oxide, vol. 7, no. 3, pp. 210-216, 2002.

[8] E. Andelová, M. Barteková, D. Pancza, J. Styk, and T. Ravingerová, "The role of NO in ischemia/reperfusion injury in isolated rat heart," General Physiology and Biophysics, vol. 24, no. 4, pp. 411-426, 2005.

[9] D. H. Endemann and E. L. Schiffrin, "Nitric oxide, oxidative excess, and vascular complications of diabetes mellitus," Current Hypertension Reports, vol. 6, no. 2, pp. 85-89, 2004.

[10] K. Naruse, C. Rask-Madsen, N. Takahara et al., "Activation of vascular protein kinase C-beta; inhibits Akt-dependent endothelial nitric oxide synthase function in obesityassociated insulin resistance," Diabetes, vol. 55, no. 3, pp. 691698, 2006.

[11] D. Vicent, J. Ilany, T. Kondo et al., "The role of endothelial insulin signaling in the regulation of vascular tone and insulin resistance," Journal of Clinical Investigation, vol. 111, no. 9, pp. 1373-1380, 2003.

[12] M. S. Sumeray, D. D. Rees, and D. M. Yellon, "Infarct size and nitric oxide synthase in murine myocardium," Journal of Molecular and Cellular Cardiology, vol. 32, no. 1, pp. 35-42, 2000.

[13] B. I. Jugdutt, "Nitric oxide and cardioprotection during ischemia-reperfusion," Heart Failure Reviews, vol. 7, no. 4, pp. 391-405, 2002.
[14] Z. Wang, M. Li, W. K. Wu, H. M. Tan, and D. F. Geng, "Ginsenoside Rb1 preconditioning protects against myocardial infarction after regional ischemia and reperfusion by activation of phosphatidylinositol-3- kinase signal transduction," Cardiovascular Drugs and Therapy, vol. 22, no. 6, pp. 443-452, 2008.

[15] L. Guan, W. Li, and Z. Liu, "Effect of ginsenoside-Rb1 on cardiomyocyte apoptosis after ischemia and reperfusion in rats," Journal of Huazhong University of Science and TechnologyMedical Science, vol. 22, no. 3, pp. 212-215, 2002.

[16] M. Thirunavukkarasu, S. V. Penumathsa, S. Koneru et al., "Resveratrol alleviates cardiac dysfunction in streptozotocininduced diabetes: role of nitric oxide, thioredoxin, and heme oxygenase," Free Radical Biology and Medicine, vol. 43, no. 5, pp. 720-729, 2007.

[17] L. N. Maslov, Y. B. Lishmanov, P. R. Oeltgen et al., "Activation of peripheral $\delta 2$ opioid receptors increases cardiac tolerance to ischemia/reperfusion injury. Involvement of protein kinase C, NO-synthase, KATP channels and the autonomic nervous system," Life Sciences, vol. 84, no. 19-20, pp. 657-663, 2009.

[18] Z. Y. Xia, J. Gao, A. K. Ancharaz, K. X. Liu, Z. Xia, and T. Luo, "Ischaemic post-conditioning protects lung from ischaemiareperfusion injury by up-regulation of haeme oxygenase-1," Injury, vol. 41, no. 5, pp. 510-516, 2010.

[19] P. Liu, J. Z. Xiang, L. Zhao, L. Yang, B. R. Hu, and Q. Fu, "Effect of $\beta 2$-adrenergic agonist clenbuterol on ischemia/reperfusion injury in isolated rat hearts and cardiomyocyte apoptosis induced by hydrogen peroxide," Acta Pharmacologica Sinica, vol. 29, no. 6, pp. 661-669, 2008.

[20] T. Nishikawa, D. Edelstein, X. L. Du et al., "Normalizing mitochondrial superoxide production blocks three pathways of hyperglycaemic damage," Nature, vol. 404, no. 6779, pp. 787-790, 2000.

[21] J. A. Beckman, A. B. Goldfine, M. B. Gordon, and M. A. Creager, "Ascorbate restores endothelium-dependent vasodilation impaired by acute hyperglycemia in humans," Circulation, vol. 103, no. 12, pp. 1618-1623, 2001.

[22] K. C. B. Tan, W. S. Chow, V. H. G. Ai, C. Metz, R. Bucala, and K. S. L. Lam, "Advanced glycation end products and endothelial dysfunction in type 2 diabetes," Diabetes Care, vol. 25, no. 6, pp. 1055-1059, 2002.

[23] C. Szabó, A. Zanchi, K. Komjáti et al., "Poly(ADP-ribose) polymerase is activated in subjects at risk of developing type 2 diabetes and is associated with impaired vascular reactivity," Circulation, vol. 106, no. 21, pp. 2680-2686, 2002.

[24] F. Cosentino, M. Eto, P. De Paolis et al., "High glucose causes upregulation of cyclooxygenase-2 and alters prostanoid profile in human endothelial cells: role of protein kinase $\mathrm{C}$ and reactive oxygen species," Circulation, vol. 107, no. 7, pp. 10171023, 2003.

[25] J. Kajstura, F. Fiordaliso, A. M. Andreoli et al., "IGF-1 0verexpression inhibits the development of diabetic cardiomyopathy and angiotensin II-mediated oxidative stress," Diabetes, vol. 50, no. 6, pp. 1414-1424, 2001.

[26] J. C. Marty, S. Bendhadra, S. Amoureux et al., "Oxidative stress is exacerbated in diabetic patients during cardiopulmonary bypass," Annales de Cardiologie et d'Angeiologie, vol. 57, no. 3, pp. 155-160, 2008.

[27] S. P. Jones, J. J. M. Greer, R. van Haperen, D. J. Duncker, R. De Crom, and D. J. Lefer, "Endothelial nitric oxide synthase overexpression attenuates congestive heart failure in mice," Proceedings of the National Academy of Sciences of the United States of America, vol. 100, no. 8, pp. 4891-4896, 2003. 
[28] A. G. Herman and S. Moncada, "Therapeutic potential of nitric oxide donors in the prevention and treatment of atherosclerosis," European Heart Journal, vol. 26, no. 19, pp. 1945-1955, 2005.

[29] B. R. Sharp, S. P. Jones, D. M. Rimmer, and D. J. Lefer, "Differential response to myocardial reperfusion injury in eNOS-deficient mice," American Journal of Physiology-Heart and Circulatory Physiology, vol. 282, no. 6, pp. H2422-H2426, 2002.

[30] F. Brunner, R. Maier, P. Andrew, G. Wölkart, R. Zechner, and B. Mayer, "Attenuation of myocardial ischemia/reperfusion injury in mice with myocyte-specific overexpression of endothelial nitric oxide synthase," Cardiovascular Research, vol. 57, no. 1, pp. 55-62, 2003.

[31] J. W. Calvert and D. J. Lefer, "Myocardial protection by nitrite," Cardiovascular Research, vol. 83, no. 2, pp. 195-203, 2009.

[32] X. P. Yang, Y. H. Liu, E. G. Shesely, M. Bulagannawar, F. Liu, and O. A. Carretero, "Endothelial nitric oxide gene knockout mice: cardiac phenotypes and the effect of angiotensinconverting enzyme inhibitor on myocardial ischemia/reperfusion injury," Hypertension, vol. 34, no. 1, pp. 24-30, 1999.

[33] J. P. Lü, Z. C. Ma, J. Yang, J. Huang, S. R. Wang, and S. Q. Wang, "Ginsenoside Rg1-induced alterations in gene expression in TNF- $\alpha$ stimulated endothelial cells," Chinese Medical Journal, vol. 117, no. 6, pp. 871-876, 2004.

[34] W. Zhou, H. Chai, P. H. Lin, A. B. Lumsden, Q. Yao, and C. Chen, "Ginsenoside Rb1 blocks homocysteine-induced endothelial dysfunction in porcine coronary arteries," Journal of Vascular Surgery, vol. 41, no. 5, pp. 861-868, 2005.

[35] W. Fu, B. S. Conklin, P. H. Lin, A. B. Lumsden, Q. Yao, and C. Chen, "Red wine prevents homocysteine-induced endothelial dysfunction in porcine coronary arteries," Journal of Surgical Research, vol. 115, no. 1, pp. 82-91, 2003.

[36] T. A. Spencer, H. Chai, W. Fu et al., "Estrogen blocks homocysteine-induced endothelial dysfunction in porcine coronary arteries," Journal of Surgical Research, vol. 118, no. 1, pp. 83-90, 2004.

[37] Z. Y. Xia, X. Y. Liu, L. Y. Zhan, Y. H. He, T. Luo, and Z. Xia, "Ginsenosides compound (shen-fu) attenuates gastrointestinal injury and inhibits inflammatory response after cardiopulmonary bypass in patients with congenital heart disease," Journal of Thoracic and Cardiovascular Surgery, vol. 130, no. 2, pp. 258-264, 2005.

[38] A. S. Attele, Y. P. Zhou, J. T. Xie et al., "Antidiabetic effects of Panax ginseng berry extract and the identification of an effective component," Diabetes, vol. 51, no. 6, pp. 1851-1858, 2002.

[39] J. Yu, M. Eto, M. Akishita, A. Kaneko, Y. Ouchi, and T. Okabe, "Signaling pathway of nitric oxide production induced by ginsenoside $\mathrm{Rb} 1$ in human aortic endothelial cells: a possible involvement of androgen receptor," Biochemical and Biophysical Research Communications, vol. 353, no. 3, pp. 764 769, 2007. 


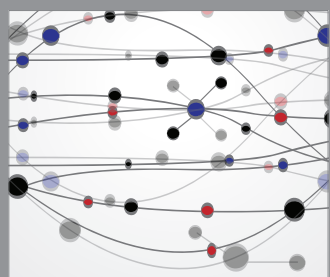

The Scientific World Journal
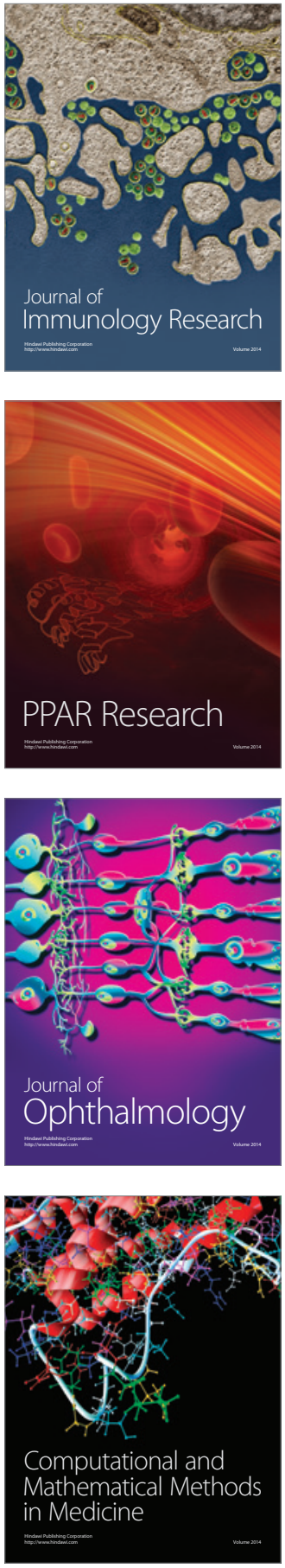

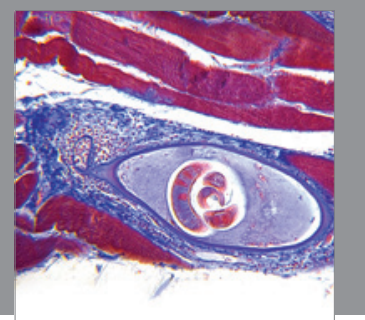

Gastroenterology

Research and Practice
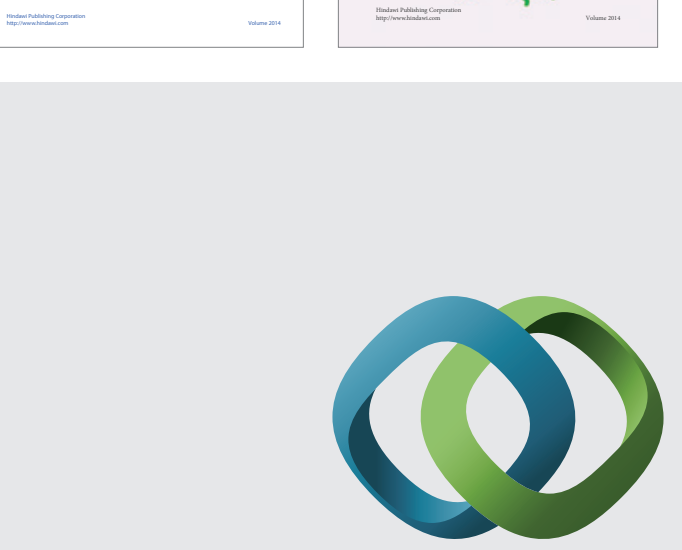

\section{Hindawi}

Submit your manuscripts at

http://www.hindawi.com
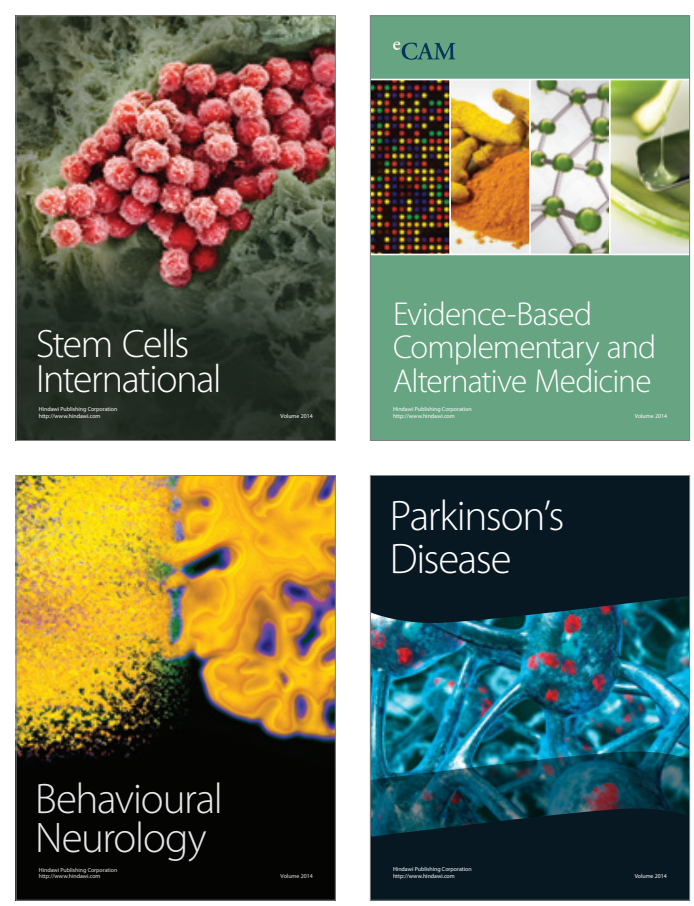

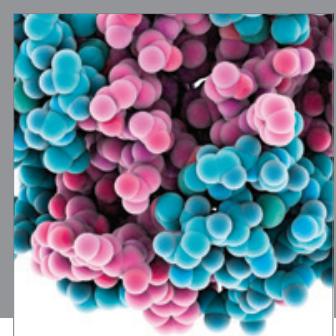

Journal of
Diabetes Research

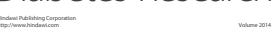

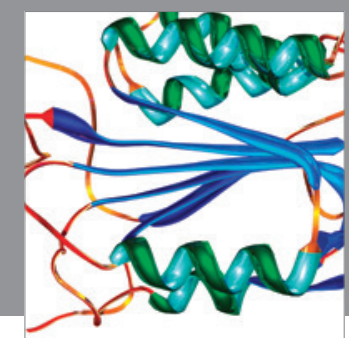

Disease Markers
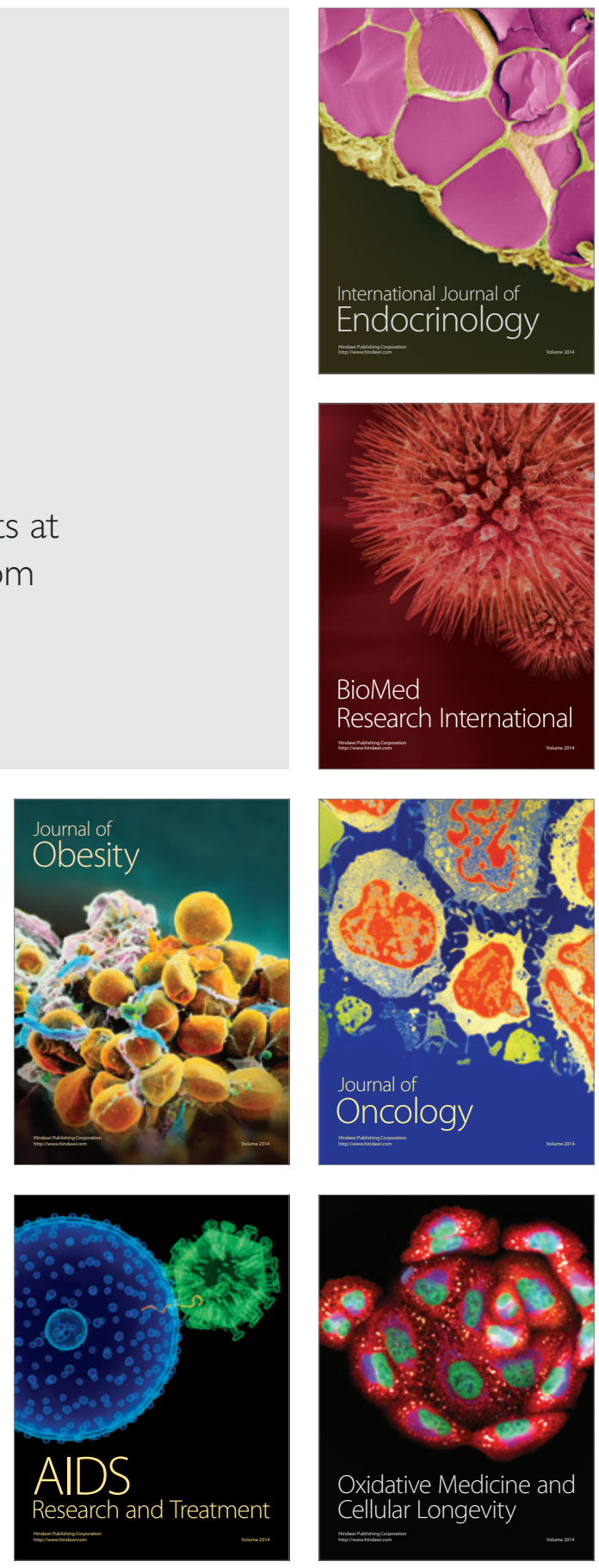